\title{
Role of Cerebellar Interpositus Nucleus in the Genesis and Control of Reflex and Conditioned Eyelid Responses
}

\author{
Lydia Jiménez-Díaz, Juan de Dios Navarro-López, Agnès Gruart, and José M. Delgado-García \\ División de Neurociencias, Universidad Pablo de Olavide, Sevilla-41013, Spain
}

The role of cerebellar circuits in the acquisition of new motor abilities is still a matter of intensive debate. To establish the contribution of posterior interpositus nucleus (PIN) to the performance and/or acquisition of reflex and classically conditioned responses (CRs) of the eyelid, the effects of microstimulation and/or pharmacological inhibition by muscimol of the nucleus were investigated in conscious cats. Microstimulation of the PIN in naive animals evoked ramp-like eyelid responses with a wavy appearance, without producing any noticeable plastic functional change in the cerebellar and brainstem circuits involved. Muscimol microinjections decreased the amplitude of reflex eyeblinks evoked by air puffs, both when presented alone or when paired with a tone as conditioned stimulus (CS). In half-conditioned animals, muscimol injections also decreased the amplitude and damped the typical wavy profile of CRs, whereas microstimulation of the same sites increased both parameters. However, neither muscimol injections nor microstimulation modified the expected percentage of CRs, suggesting a major role of the PIN in the performance of eyelid responses rather than in the learning process. Moreover, the simultaneous presentation of CS and microstimulation in well trained animals evoked CRs similar in amplitude to the added value of those evoked by the two stimuli presented separately. In contrast, muscimol-injected animals developed CRs to paired CS and microstimulation presentations, larger than those evoked by the two stimuli when presented alone. It is concluded that the PIN contributes to the enhancement of both reflex and conditioned eyelid responses and to the damping of resonant properties of neuromuscular elements controlling eyelid kinematics.

Key words: classical conditioning; cats; eyeblinks; interpositus nucleus; microstimulation; muscimol

\section{Introduction}

The neural structure(s) in which learning takes place, as well as the putative mechanisms involved, is currently a matter of intensive research and debate (Woody, 1986; Bliss and Collingridge, 1993; Malenka, 1995; Medina et al., 2002; Christian and Thompson, 2003). With respect to the classical conditioning of the nictitating membrane-eyelid response, the involvement of the cerebellar cortex and/or the underlying deep nuclei in the acquisition and storage of these learned motor responses has been suggested (Llinás and Welsh, 1993; Carey and Lisberger, 2002; Christian and Thompson, 2003). In particular, some not yet well defined regions of the anterior and/or posterior interpositus nuclei (PIN) have been proposed to be the sites involved in this type of associative learning (Krupa et al., 1993; Mauk, 1997; Bracha et al., 2001). Indeed, specific areas of the interpositus nucleus are involved in eyelid responses, as recently confirmed by retrograde transneuronal tracing with rabies virus in rats (Morcuende et al., 2002), lesions and pharmacological studies in rabbits and cats (Yeo et al., 1985; Krupa et al., 1993; Hardi-

Received May 26, 2004; revised July 26, 2004; accepted Aug. 20, 2004.

This work was supported by grants from the Spanish Ministerio de Ciencia y Tecnología (MCYT) (BFI2002-00936), Fundació La Caixa (00/032-00), and Junta de Andalucía (CVI-122). L.J.-D. and J.D.N.-L. are fellows of the Instituto de Salud Carlos III and MCYT of Spain. We thank R. Sánchez-Campusano for computer assistance and Roger Churchill for editorial help.

Correspondence should be addressed to Prof. José M. Delgado-García, División de Neurociencias, Universidad Pablo de Olavide, Carretera de Utrera, Km 1, Sevilla-41013, Spain. E-mail: jmdelgar@dex.upo.es.

DOI:10.1523/JNEUROSCI.2025-04.2004

Copyright $\odot 2004$ Society for Neuroscience $\quad$ 0270-6474/04/249138-08\$15.00/0 man et al., 1996; Mauk, 1997; Bracha et al., 1999; Christian and Thompson, 2003), and unitary recordings in conscious cats (Gruart et al., 2000).

A still-open question refers to the contribution of cerebellar circuits to eyelid conditioned responses (CRs). On the one hand, many lesion and pharmacological studies suggest that the interpositus nuclei could be the site for this motor learning (Krupa et al., 1993; Mauk, 1997; Bracha et al., 2001), although others have suggested that the cerebellum is mostly involved in the performance of CRs (Welsh and Harvey, 1989, 1991; Welsh, 1992; Gruart et al., 2000; Delgado-García and Gruart, 2002; Seidler et al., 2002) or in their proper timing (Garcia and Mauk, 1998; Koekkoek et al., 2003), but not in the acquisition itself. Moreover, electrophysiological recordings of selected cerebellar nuclei sites related to eyelid kinematics (Morcuende et al., 2002) have shown that the firing of identified nuclei neurons lag (rather than precede) the initiation of reflex and conditioned eyelid responses, at least in cats (Gruart and Delgado-García, 1994; Gruart et al., 2000), suggesting that the cerebellum is not involved in the initiation but rather in the regulation and timing of learned eyelid responses.

In the present study, we consider the contribution of the PIN to both acquisition and performance of eyelid CRs. For this aim, we performed unitary recordings of deep nuclei neurons to locate eyelid-related neural sites and implanted a guide cannula in the selected area. Cannula implantation allowed us the repeated manipulation of the same region (by microstimulation and/or 
muscimol microinjection), and because we used the magnetic search-coil technique for the eyelid position recording over time, we were able to relate manipulations of the PIN to the acquisition of eyelid CRs, affecting either their percentage or their kinematics, timing, and oscillatory properties. Results indicated that facilitation (by microstimulation) or disfacilitation (by muscimol injection) of PIN neurons does not modify the acquisition of eyelid CRs but greatly modifies their performance, oscillatory properties, timing, and internal structure.

\section{Materials and Methods}

Subjects. Experiments were performed on nine adult female cats weighing 2.1-2.7 kg. Animals were obtained from an authorized supplier (IFFA Credo, Arbresle, France). All of the experiments were performed in accordance with guidelines of the European Union (86/609/EU) for the use of laboratory animals in chronic experiments.

Surgical procedures. Animals were anesthetized with sodium pentobarbital $(35 \mathrm{mg} / \mathrm{kg}$, i.p.) after an injection of atropine sulfate $(0.5 \mathrm{mg} / \mathrm{kg}$, i.m.) to prevent unwanted vagal reflexes. Animals were implanted with a search coil (five turns; $3 \mathrm{~mm}$ in diameter) in the center of the left upper eyelid, at $2 \mathrm{~mm}$ from the lid margin (see Fig. $1 A$ ). The coil was made from Teflon-coated multistranded stainless steel wire, with an external diameter of $50 \mu \mathrm{m}$. Eyelid movements were recorded with the magnetic search-coil technique (Gruart et al., 1995). Animals were also implanted with two hook electrodes, bared $1 \mathrm{~mm}$ at their tips, in the ipsilateral orbicularis oculi muscle, for electromyographic (EMG) recordings. Recording electrodes were made of the same wire. A stimulating bipolar (200 $\mu \mathrm{m}$ enamel-coated silver wire) electrode was implanted in the magnocellular division of the contralateral red nucleus for the antidromic activation of recorded interpositus neurons. A recording window $(5 \times 5$ $\mathrm{mm}$ ) was opened in the occipital bone to allow access to the interpositus nucleus. The dura mater was removed, and an acrylic chamber was constructed around the opening. The cerebellar surface was protected with a piece of silicone sheet and sterile gauze and capped with a hermetic plastic cover. A head-holding system was attached to the skull at stereotaxic coordinates. Wires were soldered to a socket fixed to the holding system. A more detailed description of this chronic preparation was published previously (Gruart et al., 1995, 2000).

Guide cannula implantation. Recording sessions started 2 weeks after surgery. Deep cerebellar nuclei were explored with glass microelectrodes ( $2 \mathrm{M} \mathrm{NaCl} ; 3-5 \mathrm{M} \Omega$ of resistance) to locate the interpositus area related to eyelid responses (Gruart et al., 2000). Once this area was found, a guide cannula consisting of a 21 gauge stainless steel needle was implanted to a depth of up to $1 \mathrm{~mm}$ over the selected site. The cannula was maintained sterile and protected by a removable 25 gauge stainless steel rod during nonrecording periods. After cannula implantation, the cerebellar surface was protected with a sterile silicone sheet, and the chamber was sealed with acrylic cement.

Reflexively evoked blinks. Three of the animals were selected at random for the study of air-puff-evoked eyelid responses during microstimulation of, or after muscimol injection into, the PIN. Recording sessions lasted $<2 \mathrm{hr}$, for a maximum of $20 \mathrm{~d}$. Reflex eyelid responses were evoked by an unconditioned stimulus (US) that consisted of air puffs (100 msec; $3 \mathrm{~kg} / \mathrm{cm}^{2}$ ) directed to the ipsilateral cornea.

Classical conditioning. Six of the animals were selected for conditioning: three were assigned for microstimulation studies and the other three for muscimol injections. Conditioning sessions started 2-3 d after the guide cannula was implanted. Conditioning consisted of 4 habituation and 10 conditioning sessions. A delay (tone-air puff) conditioning paradigm was used. The conditioned stimulus (CS) consisted of a $370 \mathrm{msec}$, $600 \mathrm{~Hz}, 90 \mathrm{~dB}$ tone. The US started $270 \mathrm{msec}$ after CS onset and consisted of a $100 \mathrm{msec}, 3 \mathrm{~kg} / \mathrm{cm}^{2}$ air puff directed to the left cornea (the same as that presented during reflexively evoked eyelid responses). The US coterminated with the CS. A conditioning session consisted of 12 blocks separated by a variable $(5 \pm 1 \mathrm{~min})$ interval. Each block consisted of 10 trials separated by intervals of $30 \pm 10 \mathrm{sec}$. The CS was presented alone in the first trial of each block. A complete conditioning session lasted for $\sim 2$ hr. The CS was presented alone during habituation sessions for the same number of blocks per session and trials per block. As criterion, we considered a CR to be those eyelid movements appearing in response to CS presentation with $>3$ degrees (deg) of amplitude and a latency of $>40 \mathrm{msec}$, and in absence of any other eyelid response in the preceding $200 \mathrm{msec}$.

Electrical stimulation and microinjections. Microstimulations and muscimol injections performed during conditioning sessions were similar to those during reflexively evoked eyelid responses. Data were collected $1 \mathrm{~d}$ after the 10th conditioning session (see Fig. 6).

For microstimulation, we used cathodal, square pulses of $50 \mu \mathrm{sec}$ and $50 \mu \mathrm{A}$. Pulses were presented at 1 or $20 \mathrm{~Hz}$. Trains of $20 \mathrm{~Hz}$ pulses never lasted $>1 \mathrm{sec}$. These stimulation patterns were selected according to the indications of a previous study (Gruart et al., 2000). Microstimulation was performed with the help of homemade, bipolar, concentric, stainless steel electrodes with an external diameter of 25 gauge and a resistance of $1-1.5 \mathrm{M} \Omega$. The stimulating electrode was allowed to project $1.5 \mathrm{~mm}$ beyond the guide cannula tip. When presented simultaneously with the CS, the train of stimuli was set to start $40 \mathrm{msec}$ after the beginning of the tone.

Injections of muscimol (Sigma, St. Louis, MO) were made with the help of a Hamilton syringe $(1 \mu \mathrm{l})$ connected by a calibrated plastic tube to a 25 gauge stainless steel needle inserted inside the guide cannula. Injections were performed at a rate of $0.25 \mu \mathrm{l} / \mathrm{min}$. In total, $1.25 \mu \mathrm{g} / \mathrm{kg}$ of muscimol dissolved in $1 \mu \mathrm{l}$ of artificial CSF, pH 7.4, was injected $20 \mathrm{~min}$ before conditioning sessions.

Histology. At the end of the experiments, animals were deeply reanesthetized $(50 \mathrm{mg} / \mathrm{kg}$ sodium pentobarbital, i.p.). Electrolytic marks were placed at the recording, injection, and microstimulation sites with the help of a tungsten microelectrode ( $1 \mathrm{~mA}$ for $30 \mathrm{sec})$. Animals were perfused transcardially with saline and phosphate-buffered formalin. Serial sections $(50 \mu \mathrm{m})$ of the cerebellum were mounted on glass slides and stained with toluidine blue, for confirmation of stimulating and recording sites.

Data collection and analysis. Eyelid position, EMG and neuronal activity, and $1 \mathrm{~V}$ rectangular pulses corresponding to CS and US presentations were stored digitally on a computer using an analog-digital converter (CED 1401 Plus; Ceta Electronic Design, Cambridge, UK), at a sampling frequency of $11-22 \mathrm{kHz}$ and an amplitude resolution of $12 \mathrm{bits}$. Commercial computer programs (Spike 2 and SIGAVG; Ceta Electronic Design) were modified to represent instantaneous firing rate, EMG recordings, and eyelid position and velocity. The instantaneous firing rate was calculated as the inverse of the interspike intervals (see Fig. 1C). Velocity and acceleration profiles were computed digitally as the first and second derivatives of eyelid position records after low-pass filtering of the data $(-3 \mathrm{~dB}$ cutoff at $50 \mathrm{~Hz}$, with a 0 at $\approx 100 \mathrm{~Hz}$ ). Data were processed for statistical analysis using the SPSS for Windows package. Unless indicated otherwise, data are represented by the mean \pm SEM. The power of the spectral density function (i.e., the power spectrum) of selected segments from eyelid acceleration records was calculated using a fast Fourier transform to define the relative strength of the different frequencies present in eyelid responses. Significance of power spectra peaks was tested with the $\chi^{2}$-distributed test for spectral density functions (Gruart et al., 1995; Domingo et al., 1997). Collected data were analyzed using ANOVA with time or session as repeated measure, coupled with contrast analysis when appropriate. Singular differences between control and muscimol groups were analyzed with Student's $t$ test.

\section{Results}

During preliminary sessions, the deep cerebellar nuclei were systematically recorded to find unitary activity related with eyelid responses. The unitary activity was recorded during the random presentation of blink-evoking stimuli (Fig. $1 A$ ). This procedure took up to eight recording sessions. In total, $135 \mathrm{U}$ recorded in cerebellar nuclear areas met the double criteria of being antidromically activated from the red nucleus and identified by collision test, and of responding to air puffs applied to the ipsilateral cornea. The location of these units, according to Berman's atlas (Berman, 1968), and as confirmed later by histological procedures, was as follows: $2 \%$ of the neurons were located at fastigial nucleus, $18 \%$ at anterior interpositus nucleus, $57 \%$ at PIN, and 

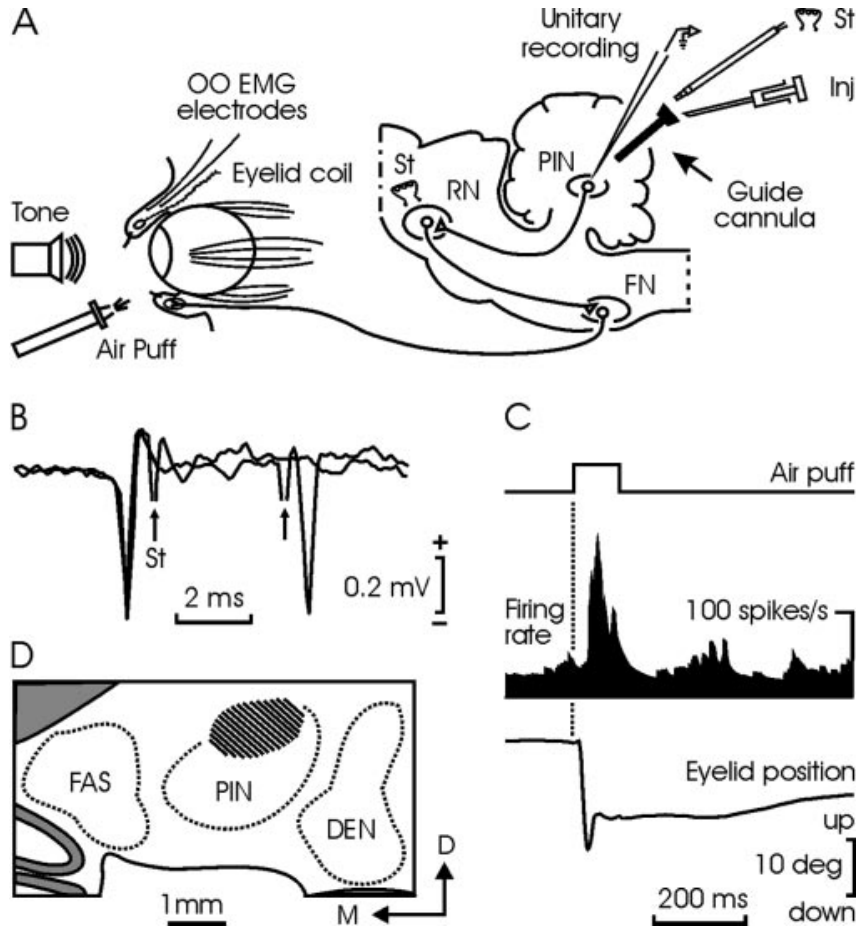

Figure 1. Experimental design. $A$, Cats were presented with air puffs and tones to evoke reflex and conditioned eyelid responses. The locations of implanted eyelid coils and orbicularis oculi electromyographic (00 EMG) electrodes are shown. Ipsilateral PIN neurons were identified by their antidromic activation from the contralateral red nucleus (RN). A guide cannula was implanted in the selected site to allow microinjection (Inj) and microstimulation (St) of PIN neurons. $B$, Two superimposed traces illustrate the collision between spontaneous spikes from a PIN neuron and those evoked antidromically by the electrical stimulation (arrows) of the RN.C, Firing rate of the neuron in $B$ during an air-puff-evoked blink. $D, A$ coronal view of studied PIN sites (dashed area), corresponding to the plane P9 of Berman's atlas (Berman, 1968). DEN, Dentate; FAS, fastigial nuclei; FN, facial nucleus; D, dorsal; M, medial.

$23 \%$ at dentate nucleus. Because of their firing during spontaneous and reflex eyelid responses, and in accordance with previous reports (Van Kan et al., 1993; Gruart and Delgado-García, 1994), the larger number of recorded neurons (98 of 135) was classified as type A (i.e., those firing during active blinking), whereas the others (37 of 135) were classified as type B (i.e., those with a sustained firing that decreases to a stop during downward eyelid responses).

For all of the animals $(n=9)$, and as confirmed by the histological study, the dorsomedial part of the PIN was found to contain the densest concentration of neurons (76 of 135) related to reflex eyelid responses (Fig. 1D), and it was chosen for developing the rest of the experiments. Posterior interpositus type A neurons ( 55 of 76 ) were identified by their antidromic activation $(0.57-0.81 \mathrm{msec})$ from the contralateral red nucleus (Fig. $1 B$ ). Neuronal firing increased during downward movements of the upper eyelid, lagging lid displacement by $>10 \mathrm{msec}$ (Fig. 1C). Neuronal discharge rates were related either to eyelid position (gain $0.5-8.0$ spikes/deg; $r \geq 0.8 ; p \leq 0.05$ ) or to eyelid velocity $\left(0.06-0.20\right.$ spikes $\left.\cdot \mathrm{deg}^{-1} \cdot \mathrm{sec}^{-1} ; r \geq 0.8 ; p \leq 0.05\right)$. Posterior interpositus type B neurons ( 21 of 76 ) were also antidromically activated from the red nucleus $(0.81-1.20 \mathrm{msec})$ and presented a noticeable inhibition in their firing that was coincidental with the downward displacement of the upper eyelid during reflexively evoked blinks (Gruart and Delgado-García, 1994). Finally, once the area of study had been selected, the guide cannula was implanted and the designed experiments were started.
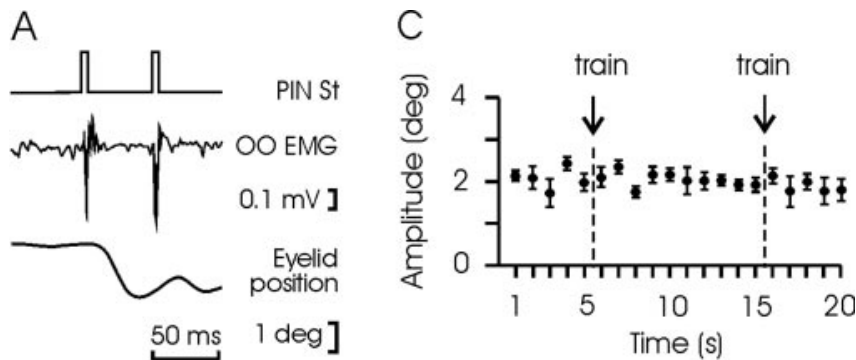

B

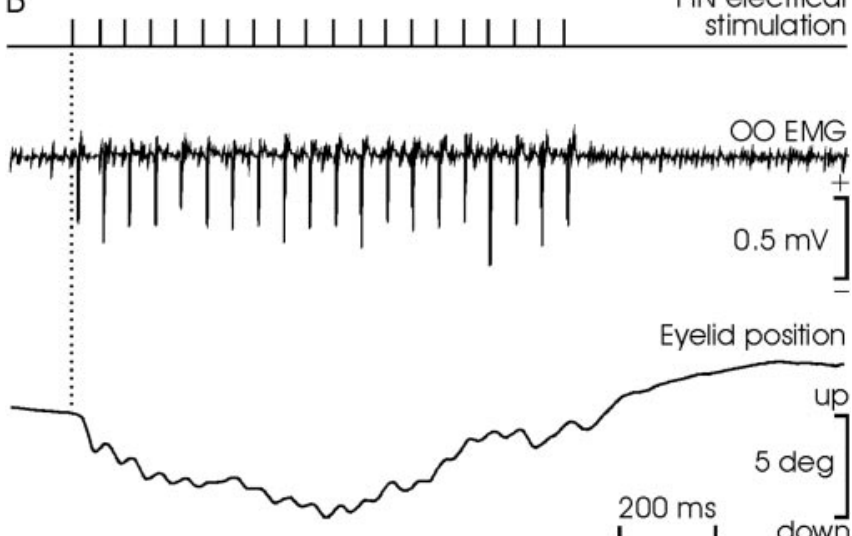

Figure 2. Eyelid responses evoked by microstimulation of the PIN. $A$, Orbicularis oculi electromyography (00 EMG) and eyelid response to two electrical pulses $(50 \mu \mathrm{sec} ; 50 \mu \mathrm{A})$ applied to the ipsilateral PIN. Note the small amplitude of the evoked eyelid response. $B, 00 \mathrm{EMG}$ and eyelid responses evoked by a train $(20 \mathrm{~Hz})$ of electrical pulses applied to the PIN. C, Trains of stimuli $(20 \mathrm{~Hz}$ for $1 \mathrm{sec}$ ) applied to the PIN had no long-lasting effects (ANOVA) on eyelid responses (mean amplitude $\pm \mathrm{SEM} ; n=10$ ) evoked by single pulses applied to the same site. The single pulses were applied at $1 \mathrm{~Hz}$, and the train was presented twice as indicated (arrows). St, Electrical stimulation.

As illustrated in Figure $2 B$, microstimulation of the PIN at 20 $\mathrm{Hz}$ for $1 \mathrm{sec}$ evoked a ramp-like downward displacement of the upper eyelid more similar in its profile to a CR than to a reflex blink (Gruart et al., 1995). Each single pulse evoked an eyelid displacement lasting for $\approx 50 \mathrm{msec}$, with amplitudes ranging from 0.5 to 2 deg (i.e., $1 / 40-1 / 10$ of maximal eyelid response) and a peak velocity of $40-180 \mathrm{deg} / \mathrm{sec}$ (Fig. 2A). Given their small amplitude, eyelid displacements evoked by PIN microstimulation produced minor modifications in air-puff-evoked blinks (data not shown). The trains of stimuli applied at the PIN did not evoke any long-term potentiation (or depression) of the motor effects evoked by a single stimulus presented at $1 \mathrm{~Hz}$ (Fig. 2C). Accordingly, the effects of PIN microstimulation on reflex and conditioned eyelid responses described below cannot be ascribed to plastic changes evoked in the cerebellar and brainstem circuits involved.

A single injection of muscimol $(1.25 \mu \mathrm{g} / \mathrm{kg})$ in the same PIN site produced a decrease (of up to $20 \%$ of the initial response) in the amplitude of air-puff-evoked blinks (Fig. 3A). Reflex blinks were significantly $(p<0.001)$ smaller $(34 \% ; n=5$; in three animals) $5 \mathrm{~min}$ after the injection. The effects of muscimol were still clearly noticeable $(93 \% ; p<0.001) 70 \mathrm{~min}$ after its injection but were completely removed after $24 \mathrm{hr}$ (Fig. 3B). Interestingly, muscimol had no significant effect on the latency of reflexively evoked blinks. Muscimol also decreased the amplitude of eyelid responses evoked by US when this was applied as part of a CS-US pair presentation (i.e., when the air puff was paired with a tone as a CS). As illustrated in Figure 3, $C$ and $D$, the normalized ampli- 
A

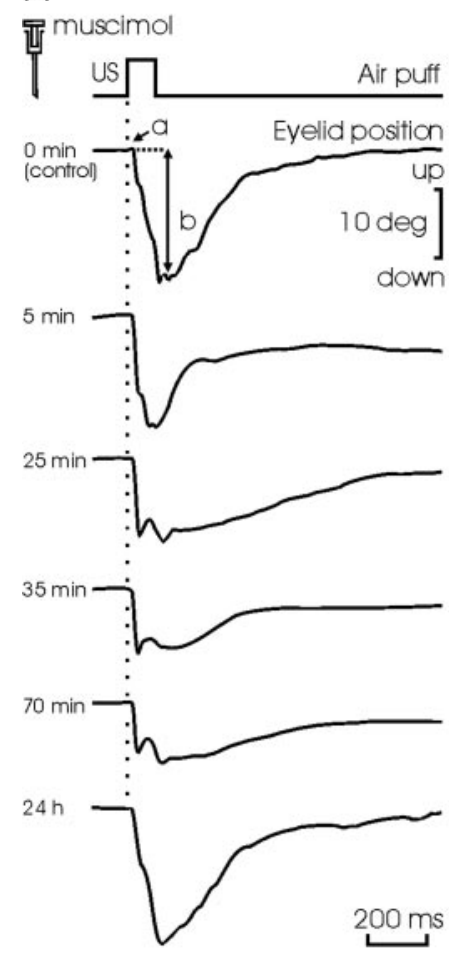

C
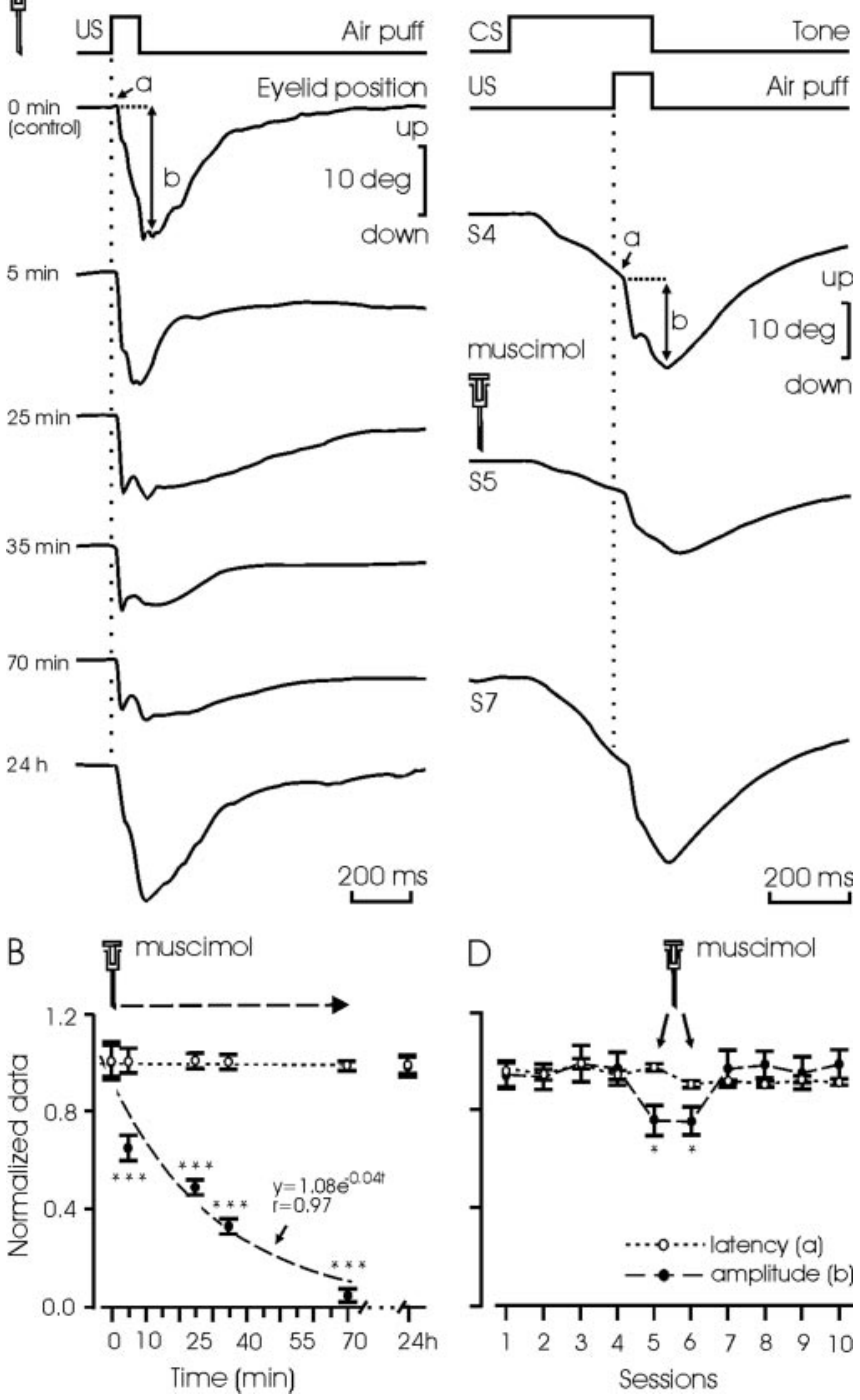

Figure 3. Effects of muscimol on reflex eyelid responses. $A$, Effects of a single injection of muscimol into the posterior interpositus nucleus $(1.25 \mu \mathrm{g} / \mathrm{kg})$, on the latency (a) and amplitude (b) of air-puff-evoked ( $100 \mathrm{msec} ; 3 \mathrm{~kg} / \mathrm{cm}^{2}$ ) eyelid responses. Time after drug injection is indicated. $B$, Quantitative data from the experiment in $A$, collected ( $n=5$ times) from the three nonconditioned animals. Data were normalized by dividing each collected value by the corresponding value obtained at 0 min. Means \pm SEM latency (unfilled circles and dotted line) and amplitude (filled circles and dashed line) of evoked eyelid responses are indicated. Note that the amplitude of air-puff-evoked eyelid responses was completely recovered $24 \mathrm{hr}$ after muscimol injection. C, Latency (a) and amplitude (b) of reflex eyelid responses to the same air puff used in $A$ and $B$, but presented as part of a CS-US pair, after the injection of muscimol $(1.25 \mu \mathrm{g} / \mathrm{kg})$. The drug was injected $20 \mathrm{~min}$ before the fifth conditioning session (S5). D, Quantitative data from the experiment in C, collected from three animals. Muscimol was injected before the fifth and sixth conditioning sessions. Data were normalized by dividing each collected value by the maximum value obtained in any of the conditioning sessions. Note a significant decrease in the amplitude of the evoked eyelid response (filled circles and dashed line), but not in its latency (unfilled circles and dotted line). ${ }^{*} p<0.05 ;{ }^{* * *} p<0.001$ (ANOVA).

tude of unconditioned eyelid responses decreased by $20 \%$ ( $p<$ 0.05 ) during the two conditioning sessions (fifth and sixth) in which muscimol was injected ( $n=3$ animals). Here again, muscimol did not produce any change in the latency of unconditioned responses.

We also studied the effects of PIN muscimol injection and microstimulation on the performance of eyelid CRs. As shown in
A

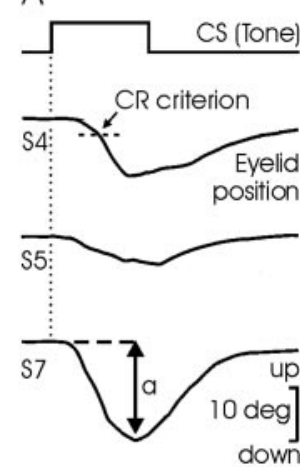

C

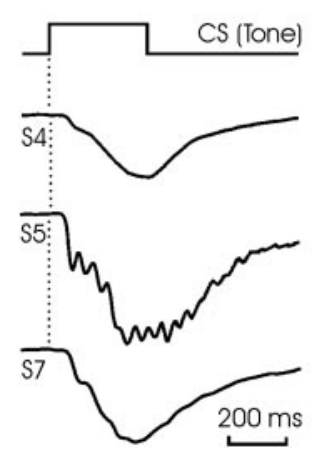

B

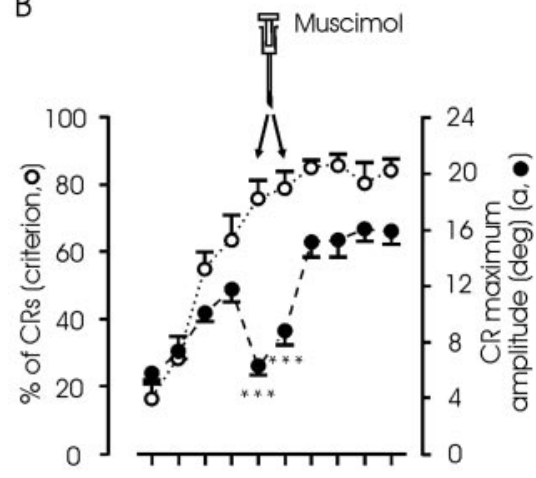

D

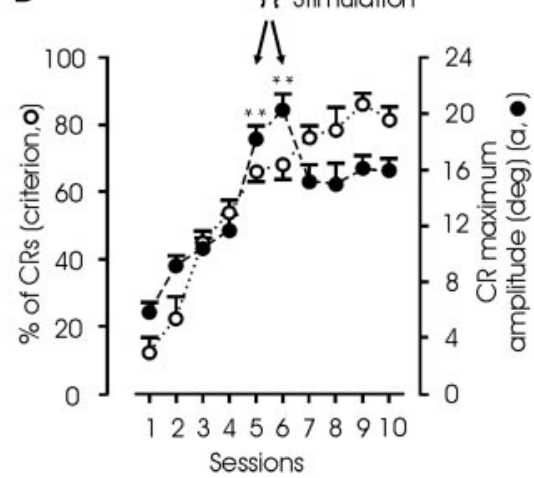

Figure 4. Effects of muscimol or microstimulation on the percentage and amplitude of CRs. $A$, Representative examples of CRs evoked by the presentation of the CS alone, collected from the fourth, fifth, and seventh conditioning sessions. Muscimol $(1.25 \mu \mathrm{g} / \mathrm{kg})$ was injected 20 min before the fifth session. $B$, Quantitative analysis of data collected from three animals (mean \pm SEM). Muscimol was injected before sessions 5 and 6 . Note that, according to the selected criterion, the expected percentage of CRs (unfilled circles and dotted line) was not modified by muscimol, but the amplitude of the evoked CRs (closed circles and dashed line) was significantly decreased (*** $p<0.001$; ANOVA). C, Representative examples of (Rs evoked by single CS presentation without (4th and 7th sessions) and with (5th session) microstimulation $(20 \mathrm{~Hz}$ for $1 \mathrm{sec}$; pulses of $50 \mu \mathrm{sec}$ and $50 \mu \mathrm{A})$ of the posterior interpositus nucleus. D, Quantitative analysis of data collected from three animals (mean \pm SEM). Microstimulation was applied during trials in which the $C S$ was presented alone. Note that, according to the selected criterion, the expected percentage of (Rs (unfilled circles and dotted line) was not modified by the microstimulation, but the amplitude of the evoked (Rs (filled circles and dashed line) was significantly increased $\left({ }^{* *} p<0.01\right.$; ANOVA).

Figure $4 A$, muscimol injection into the PIN during the fifth and sixth conditioning sessions decreased the amplitude of evoked CRs by 50\% $(p<0.001$; ANOVA $)$. However, this decrease in amplitude was not enough to produce CRs smaller than the criterion selected for the study. For this reason, the learning curve in those animals $(n=3)$ was not affected by muscimol injections (Fig. 4B, unfilled circles and dotted line). In contrast, microstimulation of the PIN increased the amplitude of the evoked CRs (Fig. 4C). In fact, the mean amplitude of CRs obtained during sessions in which microstimulation was performed (fifth and sixth) was $>150 \%$ larger $(p<0.01)$ than expected. Here again, microstimulation did not modify the percentage of CRs obtained according to the selected criterion (Fig. 4D, unfilled circles and dotted line).

A detailed analysis of CR kinematics was made for controls and after muscimol injection or electrical microstimulation (Fig. 5). A typical CR consisted of a ramp-like, downward displacement of the eyelid, with a wavy appearance (Fig. 5A). Its peak velocity was always $<200 \mathrm{deg} / \mathrm{sec}$, i.e., $10 \%$ of that reached by reflex blinks in cats (Gruart et al., 1995). The mean power spectra 
of acceleration profiles $(n=12)$ collected from CRs presented a significant $(p<$ 0.01 ) peak at $18-20 \mathrm{~Hz}$, over a broad band of frequencies between 10 and $30 \mathrm{~Hz}$ (Fig. $5 D)$. In comparison, CRs evoked after muscimol injections were $>50 \%$ smaller in amplitude $(p<0.001)$ and with a less defined power spectra profile (Fig. $5 B, D$ ). Finally, microstimulation of the PIN simultaneously with CS presentation evoked larger $(>130 \%$; $p<0.01)$ CRs than in controls, with a noticeable wavy appearance (Fig. 5C). Obviously, the mean power spectra of these CRs presented a significant peak at $20 \mathrm{~Hz}(p<$ 0.001) (Fig. 5D).

The internal structure of well defined CRs was analyzed further. The succession of small (2-3 deg in amplitude) waves that composed a CR showed no significant tendencies with respect to their duration or that of the reciprocal duration, i.e., the instantaneous frequency (data not shown) (but see Domingo et al., 1997). Within a given $\mathrm{CR}$, the successive waves decreased exponentially in peak velocity (Fig. 5E, filled circles and continuous line). The presence of muscimol damped the amplitude of these oscillations (Fig. 5D,E), whereas microstimulation of the PIN increased them (Fig. 5D,E). During microstimulation, the exponential decay in the successive peaks of velocity during a CR was still present (Fig. 5E).

The effects of combined microstimulation and muscimol injection of the PIN on the performance of CRs were also analyzed (Fig. 6). These experiments were performed on the six conditioned animals $1 \mathrm{~d}$ after the 10th conditioning session. Three of the animals were injected with muscimol, and the other three were left as controls. In this case, a total of 30 single CS presentations were performed, intermingled with a total of 90 CS-US paired presentations. Data were collected from CS-alone presentations. Microstimulations shown in Figure $6 B$ were performed at random during block intervals. As illustrated, CRs evoked in controls were larger than those evoked after the injection of muscimol $(15.1 \pm 3$ vs $7.5 \pm 2.2$ deg; $p<0.001$; Student's $t$ test) (Fig. 6 A). However, the electrical stimulation of the PIN evoked eyelid displacements of the same peak amplitude in both groups $(6.7 \pm 2.4$ vs $7.4 \pm 1.3 \mathrm{deg}$ ) (Fig. $6 \mathrm{~B}$ ). The simultaneous presentation of the CS and the PIN microstimulation evoked larger responses than the CS alone in both control and muscimol groups. In this situation, no significant difference was observed between the CRs evoked in control $(22.5 \pm 2.7 \mathrm{deg})$ and muscimol $(20.4 \pm 5.7$ deg) groups (Fig. 6C). It is important to note that the CR evoked in controls by the simultaneous presentation of the CS and the PIN microstimulation was similar in amplitude $(22.5 \mathrm{deg})$ to that of CRs evoked by the CS alone plus the microstimulation alone $(15.1+6.7=21.8 \mathrm{deg})$. In contrast, the simultaneous presentation of CS and microstimulation, in muscimol-injected animals,
B with muscimol $\quad$ C with electrical stimulation
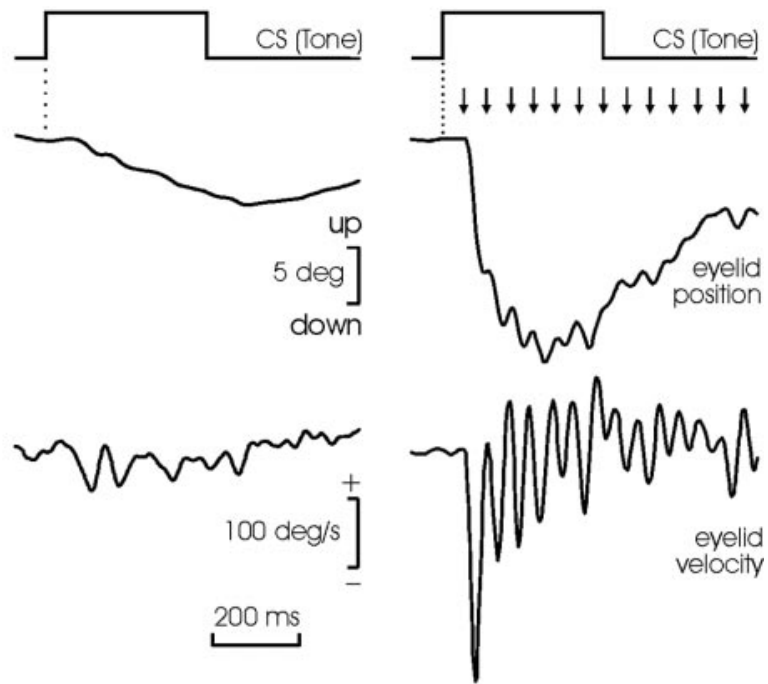

E

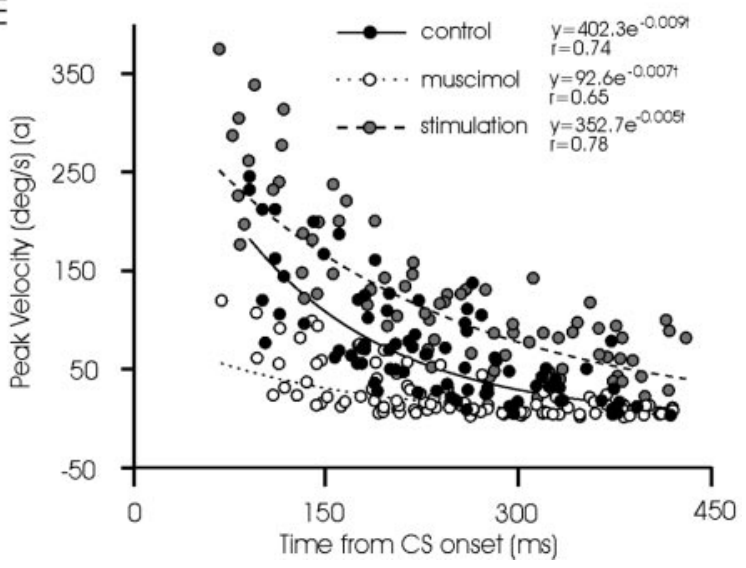

Figure 5. Frequency and peak velocity characteristics of eyelid (Rs in controls and after muscimol injection into, or microstimulation of, the PIN. $A-C$, Examples of eyelid CRs obtained by the single presentation of the $C S$ in a control animal $(A)$ and after function of time from CS presentation. Equations corresponding to the exponential line best fitting the data are shown for control (filled circles and continuous line), muscimol (unfilled circles and dotted line), and microstimulation (gray circles and dashed line) groups. Significance for fitted lines was $p<0.01$.

produced a larger CR (20.4 deg) than the aggregate value of CS and microstimulation presented alone $(7.5+7.4=14.9 \mathrm{deg} ; p<$ 0.01 ; ANOVA).

\section{Discussion}

Posterior interpositus nucleus as a site controlling reflex and learned eyelid responses

According to the present results, a very definite area of the interpositus nucleus is involved in the control and performance of both reflex and newly acquired eyelid responses. However, the PIN cannot be considered the sole place in which learned eyelid responses are generated and stored (Krupa et al., 1993; Mauk, 1997; Christian and Thompson, 2003), for the following reasons. PIN microstimulation increased CR amplitude, without changing the percentage of CRs expected in the corresponding conditioning session; thus, PIN microstimulation modified the kinetic properties of CRs, including their timing (Figs. $5 C, 6 C$ ) but not the probability of their appearance. Muscimol depression of PIN 

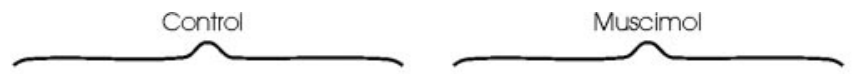

A
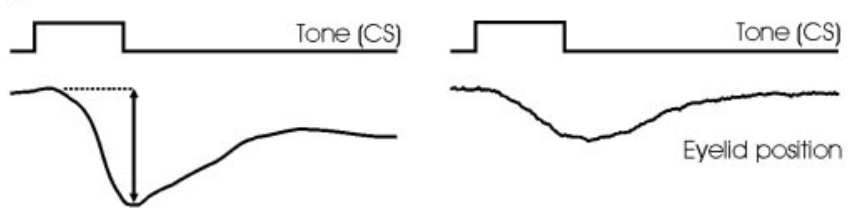

B

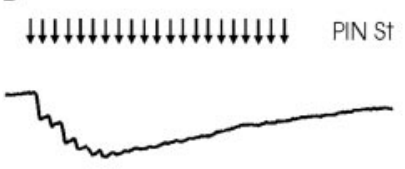

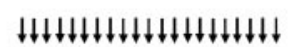

PIN S†
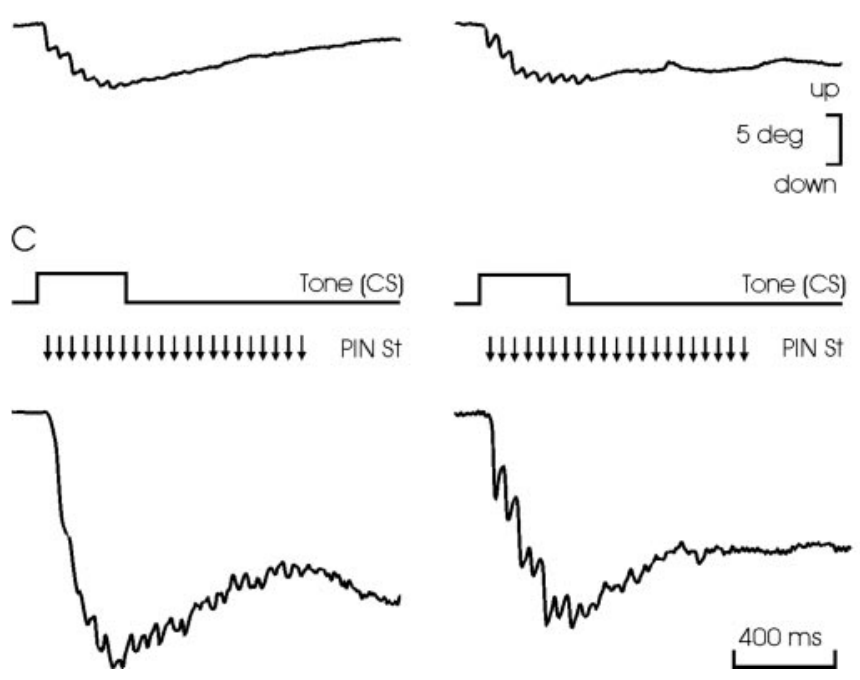

Figure 6. Effects of muscimol injection into and/or microstimulation of the PIN on eyelid CRs evoked in well trained animals. Records were performed $1 \mathrm{~d}$ after the 10th conditioning session. $A$, CRs evoked by the single presentation of the CS without (control) and after the injection of muscimol $(1.25 \mu \mathrm{g} / \mathrm{kg})$. Note the smaller $\left(n=30\right.$; ${ }^{* * *} p<0.001$; Student's $t$ test) amplitude of CRs evoked under muscimol (histograms on the right). B, Eyelid responses evoked by electrical microstimulation (St) of the PIN without (control) and after injection of the drug. Note that in this case the amplitude of evoked eyelid responses was similar in the two situations ( $n=30$; Student's $t$ test; histograms on the right). C, CRs evoked by the single presentation of the CS without (control) and after injection of the drug in the presence of the electrical microstimulation of the PIN. Note that there was no significant ( $n=30$; Student's t test) difference in the amplitudes of the evoked CRs in the two situations (histograms on the right).

activities decreased CR amplitude, without changing the expected number of CRs; moreover, and as it was pointed out previously (Welsh and Harvey, 1989; Welsh, 1992), muscimol also modified the amplitude of evoked blinks by paired and unpaired US presentations. The same results have been reported in cats and rabbits, using similar conditioning paradigms (Bracha et al., 1994, 1999), suggesting that PIN neurons are involved in the control of any kind of eyelid response and not only in those acquired by associative learning. As described previously (Gruart and Delgado-García, 1994; Gruart et al., 2000; Delgado-García and Gruart, 2002), and confirmed here for US presentations, the firing activity of PIN neurons always starts after the onset of reflex and acquired eyelid responses. The enhancer effects of the PIN on CRs can be produced, after muscimol injection, by the mere microstimulation of involved PIN neurons. In this regard, it has to be pointed out that microstimulation of PIN neurons can activate their initial axon segments even if their somatic and dendritic compartments are hyperpolarized by the action of muscimol (Llinás and Mühlethaler, 1988).

\section{Muscimol and microstimulation effects on posterior interpositus neurons on reflex and learned eyelid responses} It is generally accepted that the chemical inactivation of interpositus neurons with muscimol prevents the acquisition and ex-
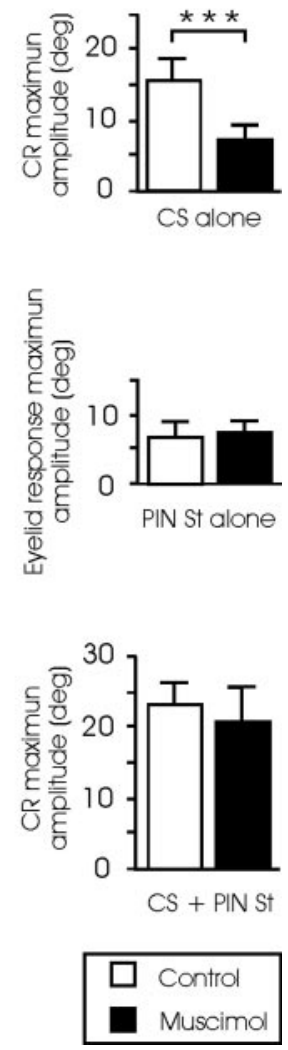

tinction of nictitating membrane-eyelid CRs (Krupa et al., 1993; Hardiman et al., 1996; Mauk, 1997; Christian and Thompson, 2003). In the present experiments, eyelid CRs were already acquired when muscimol was injected. In this situation, a significant reduction in amplitude, but not a complete abolition, of the evoked CRs was observed. A different susceptibility between rabbits (Krupa et al., 1993; Bracha et al., 1994; Hardiman et al., 1996) and cats (Bracha et al., 1999; Gruart et al., 2000) to muscimol injections in cerebellar structures has recently been proposed to explain the different strength of the CR inhibition observed in the two species (Bracha et al., 1999). The same suggestion is applicable to the effects of cerebellar nuclei lesions in rabbits compared with cats (Yu and Eidelberg, 1983; Yeo et al., 1985; Bracha et al., 1999). Moreover, the presence of even larger differences between the two species in the functional properties of their facial (Bracha et al., 1994, 1999; Gruart et al., 1995; Leal-Campanario et al., 2004) and ocular (Graf and Simpson, 1981) motor systems should be taken into account. For example, the contribution of the retractor bulbi system to conditioned eyelid-nictitating membrane responses in rabbits is extremely important, whereas accessory abducens motoneurons (i.e., the main source of innervation to the retractor bulbi muscle) do not fire at all during the performance of CRs in cats, a fact indicating that this plastic function is expressed exclusively by the facial motor nucleus in the latter species (Trigo et al., 1999; Leal-Campanario et al., 2004). Thus, the differences in cerebellar modulation of learned blink responses seen in different species should be viewed as a continuum of degrading eyeblink amplitude, rather than a quantal effect during which the acquired behavior is completely abolished by cerebellar inactivation, or not at all (Welsh and Harvey, 1989).

We found here that the deep cerebellar nuclei area more directly involved in eyelid responses corresponds to the dorsomedial part of the PIN. This finding is in agreement with previous mapping studies in cats (Gruart and Delgado-García, 1994) and monkeys (Van Kan et al., 1993). In contrast, using the lesion approach, the anterior interpositus nucleus has been related to eyeblinks in rabbits, primarily regarding the classical conditioning of the nictitating membrane response (Yeo et al., 1985; Hardiman et al., 1996; Krupa and Thompson, 1997). The unitary recordings performed here helped us to determine precisely the interpositus site involved in eyelid responses. Moreover, because axonal projections from PIN neurons run rostrally, they are always affected by lesions performed in the anterior part of the nucleus. In the same way, muscimol injections given in the latter region could easily spread to the rostral pole of the PIN. Finally, and as reported here, the use of sensitive recording techniques (Gruart et al., 1995; Koekkoek et al., 2003) to measure eyelid position is necessary to determine the presence or absence of a true CR, as well as in relation to the analysis of its proper profile, 
timing, and kinematics (Delgado-García and Gruart, 2002; Koekkoek et al., 2003).

The effect of microstimulation of the PIN, even in well trained animals, was an eyelid response that resembled a CR profile but did not reach the amplitude of a control CR. Only the combined presentation of the CS and microstimulation produced a significant increase in the amplitude of the evoked eyelid CR. Interestingly, this effect was obtained even after an injection of muscimol into the stimulated PIN. These results indicate that, in addition to the PIN, other cerebral structures need to be simultaneously active for a complete CR to occur (Delgado-García and Gruart, 2002).

Microstimulation of the PIN did not evoke any plastic change in the functional capabilities of the cerebellar and brainstem circuits involved, which were checked by single pulses applied to this structure before and after intensive train stimulation of selected PIN sites. Furthermore, single and train stimuli applied to the PIN were unable to evoke reflex-like eyelid responses, indicating that this neural structure is unable per se to initiate noticeable blinks, unless in the presence of facilitative inputs arriving at orbicularis oculi motoneurons from trigeminal (for reflex) and cortical (for learned) origins (Aou et al., 1992; Trigo et al., 1999). The presence of extracerebellar components in learned eyelid responses has also been demonstrated recently in mutant mice in which the induction of long-term depression at the parallel fiberPurkinje cell synapse is impaired (Koekkoek et al., 2003).

\section{A damping role of interpositus nucleus on eyelid responses}

According to the present results, the PIN plays an important role in damping the natural oscillatory properties of the neuromuscular elements involved in eyelid kinematics (Domingo et al., 1997; Trigo et al., 1999). Thus, muscimol injections damped the typical $\approx 20 \mathrm{~Hz}$ oscillation present in CRs, whereas microstimulation enhanced it. In fact, an oscillation of the same dominant frequency ( $\approx 20 \mathrm{~Hz}$ ) has been recorded in the EMG activity of the orbicularis oculi muscle (Domingo et al., 1997), in the membrane potential of rat facial motoneurons recorded in vitro (MagariñosAscone et al., 1999), in the discharge rate of facial nucleus motoneurons recorded in alert behaving cats (Trigo et al., 1999), and in firing activities of motor cortex neurons during performance of CRs in cats (Aou et al., 1992). In contrast, our data do not support any role of interpositus nucleus in the initiation of acquired eyelid responses, but it might participate in their proper timing with respect to the US presentation (Koekkoek et al., 2003). It can also be suggested that perhaps this function is further expanded to the overlying cerebellar cortex and/or to different cerebellar nuclear structures (e.g., the lateral part of the dentate nucleus), i.e., to areas not yet explored with this experimental approach.

According to the above, a putative role of Purkinje cell modulation of nuclear neuron firing could be to damp the natural tendency of PIN neurons to oscillate at the same frequency as the whole facial motor system. In this regard, it has been shown that some PIN neurons (named type A, as here) increase their discharge rate during the $\mathrm{CR}$, with a noticeable wide range in latencies in relation to CR onset (Gruart et al., 2000). A sequential and out-of-phase release of the activity of type A neurons will certainly be helpful for a proper performance of consolidated eyelid CRs. A similar damping role for interpositus neurons during arm-reaching responses was suggested previously, based on data collected from behaving monkeys (Thach et al., 1992).

In summary, results obtained by subjecting the PIN to microstimulation and muscimol injections helped us to understand the data collected previously with unitary recordings (Gruart and Delgado-García, 1994; Gruart et al., 2000) in the same area, demonstrating an enhancer role of interpositus neurons in the performance and proper timing of eyelid CRs (Welsh and Harvey, 1991; Delgado-García and Gruart, 2002; Seidler et al., 2002; Koekkoek et al., 2003) but not in their initiation and/or the storage of acquired memories.

\section{References}

Aou S, Woody CD, Birt D (1992) Changes in the activity of units of the cat motor cortex with rapid conditioning and extinction of a compound eyeblink movement. J Neurosci 12:549-559.

Berman AL (1968) The brain stem of the cat. A citoarchitectonic atlas with stereotaxic coordinates. Madison, WI: University of Wisconsin.

Bliss TVP, Collingridge GLA (1993) A synaptic model of memory: longterm potentiation in the hippocampus. Nature 361:31-39.

Bracha V, Webster ML, Winters NK, Irwin KB, Bloedel JR (1994) Effects of muscimol inactivation of the cerebellar interposed-dentate nuclear complex on the performance of the nictitating membrane response in the rabbit. Exp Brain Res 100:453-468.

Bracha V, Kolb FP, Irwin KB, Bloedel JR (1999) Inactivation of interposed nuclei in the cat: classically conditioned withdrawal reflexes, voluntary limb movements and the action primitive hypothesis. Exp Brain Res 126:77-92.

Bracha V, Zhao L, Irwin K, Bloedel JR (2001) The human cerebellum and associative learning: dissociation between the acquisition, retention and extinction of conditioned eyeblinks. Exp Brain Res 136:41-49.

Carey MR, Lisberger SG (2002) Behavioral analysis of gain control for smooth pursuit eye movements. Neuron 35:223-226.

Christian KM, Thompson RF (2003) Neural substrates of eyeblink conditioning: acquisition and retention. Learn Mem 10:427-455.

Delgado-García JM, Gruart A (2002) The role of interpositus nucleus in eyelid conditioned responses. Cerebellum 1:289-308.

Domingo JA, Gruart A, Delgado-García JM (1997) Quantal organization of reflex and conditioned eyelid responses. J Neurophysiol 78:2518-2530.

Garcia KS, Mauk MD (1998) Pharmacological analysis of cerebellar contributions to the timing and expression of conditioned eyelid responses. Neuropharmacology 37:471-480.

Graf W, Simpson JI (1981) Relations between the semicircular canals, the optic axis, and the extraocular muscles in the lateral-eyed and frontaleyed animals. In: Progress in oculomotor research (Fuchs AF, Becker W, eds), pp 409-417. Amsterdam: Elsevier/North-Holland.

Gruart A, Delgado-García JM (1994) Discharge of identified deep cerebellar nuclei neurons related to eye blinks in the alert cat. Neuroscience 61:665-681.

Gruart A, Blázquez P, Delgado-García JM (1995) Kinematics of spontaneous, reflex, and conditioned eyelid movements in the alert cat. J Neurophysiol 74:226-248.

Gruart A, Guillazo-Blanch G, Fernández-Mas R, Jiménez-Díaz L, DelgadoGarcía JM (2000) Cerebellar posterior interpositus nucleus as an enhancer of classically conditioned eyelid responses in alert cats. J Neurophysiol 84:2680-2690.

Hardiman MJ, Ramnani N, Yeo CH (1996) Reversible inactivations of the cerebellum with muscimol prevent the acquisition and extinction of conditioning nictitating membrane responses in the rabbit. Exp Brain Res 2:235-247.

Koekkoek SK, Hulscher HC, Dortland BR, Hensbroek RA, Elgersma Y, Ruigrok TJ, De Zeeuw CI (2003) Cerebellar LTD and learning-dependent timing of conditioned eyelid responses. Science 301:1736-1739.

Krupa DJ, Thompson RF (1997) Reversible inactivation of the cerebellar interpositus nucleus completely prevents acquisition of the classically conditioned eye-blink responses. Learn Mem 3:545-556.

Krupa DJ, Thompson JK, Thompson RF (1993) Localization of a memory trace in the mammalian brain. Science 260:989-991.

Leal-Campanario R, Barradas-Bribiescas JA, Delgado-García JM, Gruart A (2004) Relative contributions of eyelid and eye-retraction motor systems to reflex and classically conditioned blink responses in the rabbit. J Appl Physiol 96:1541-1554.

Llinás R, Mühlethaler M (1988) Electrophysiology of guinea-pig cerebellar nuclear cells in the in vitro brain stem-cerebellar preparation. J Physiol (Lond) 404:241-258. 
Llinás R, Welsh JP (1993) On the cerebellum and motor learning. Curr Opin Neurobiol 3:958-965.

Magariños-Ascone C, Núñez A, Delgado-García JM (1999) Different discharge properties of dorsolateral facial nucleus motoneurons: intracellular in vitro recordings. Neuroscience 94:879-886.

Malenka RC (1995) LTP and LTD: dynamic and interactive processes of synaptic plasticity. Neuroscientist 1:35-42.

Mauk MD (1997) Roles of cerebellar cortex and nuclei in motor learning: contradictions or clues? Neuron 18:343-346.

Medina JF, Christopher Repa J, Mauk MD, LeDoux JE (2002) Parallels between cerebellum- and amygdala-dependent conditioning. Nat Rev Neurosci 3:122-131.

Morcuende S, Delgado-García JM, Ugolini G (2002) Neuronal premotor networks involved in eyelid responses: retrograde transneuronal tracing with rabies virus from the orbicularis oculi muscle in the rat. J Neurosci 22:8808-8818.

Seidler RD, Purushotham A, Kim SG, Ugurbil K, Willingham D, Ashe J (2002) Cerebellum activation associated with performance change but not motor learning. Science 296:2043-2046.

Thach WT, Goodkin HP, Keating JG (1992) The cerebellum and the adaptive coordination of movements. Annu Rev Neurosci 15:403-442.
Trigo JA, Gruart A, Delgado-García JM (1999) Discharge profiles of abducens, accessory abducens, and orbicularis oculi motoneurons during reflex and conditioned blinks in alert cats. J Neurophysiol 81:1666-1684.

Van Kan PLE, Houk JC, Gibson AR (1993) Output organization of intermediate cerebellum of the monkey. J Neurophysiol 69:57-73.

Welsh JP (1992) Changes in the motor pattern of learned and unlearned responses following cerebellar lesions: a kinematic analysis of the nictitating membrane reflex. Neuroscience 47:1-19.

Welsh JP, Harvey JA (1989) Cerebellar lesions and the nictitating membrane reflex: performance deficits of the conditioned and unconditioned response. J Neurosci 9:299-311.

Welsh JP, Harvey JA (1991) Pavlovian conditioning in the rabbit during inactivation of the interpositus nucleus. J Physiol (Lond) 444:459-480.

Woody CD (1986) Understanding the cellular basis of memory and learning. Annu Rev Psychol 37:433-493.

Yeo CH, Hardiman MJ, Glickstein M (1985) Classical conditioning of the nictitating membrane response of the rabbit. I. Lesions of the cerebellar nuclei. Exp Brain Res 60:87-98.

Yu J, Eidelberg E (1983) Recovery of locomotor function in cats after localized cerebellar lesions. Brain Res 273:121-131. 